\title{
ERRATUM TO: ALGÈBRES DE LIE QUASI-RÉDUCTIVES
}

\author{
MICHEL DUFLO \\ Université Denis Diderot-Paris 7 \\ Institut de Mathématiques \\ de Jussieu \\ UFR de Mathématiques, Case 7012 \\ F-75205 Paris cedex 13, France \\ duflo@math.jussieu.fr
}

\author{
MOHAMED SALAH KHALGUI
}

Université Tunis El-Manar

Faculté des Sciences de Tunis

Département de Mathématiques

Campus universitaire 2092 El-Manar, Tunis, Tunisie

mohamedsalah.khalgui@fst.rnu.tn

\section{PIERRE TORASSO}

\author{
Université de Poitiers \\ Laboratoire de Mathématiques \\ et Applications \\ UMR CNRS 6086 \\ SP2MI, BP30179 \\ F-86962 Chasseneuil cedex, France \\ pierre.torasso@math.univ-poitiers.fr
}

\begin{abstract}
In Corollary 12(ii) and Theorem 13(v) of [1] we omitted the hypothesis $\operatorname{dim} \mathfrak{z} \leq 1$. Moreover, in some places the symbol $\mathbb{K}$ must be replaced by the symbol $\mathbb{K}^{\times}$.

\section{Erratum to: Transformation Groups (2012) 17: 417-470 DOI: $10.1007 / \mathrm{s} 00031-012-9179-4^{*}$}

1. In Corollary 12(ii) of [1] we omitted the hypothesis $\operatorname{dim} \mathfrak{z} \leq 1$. It should be read:

(ii) On suppose $\operatorname{dim} \mathfrak{z} \leq 1$. Alors un élément $\mu$ de $\mathfrak{z}^{*}$ est préhomogne si et seulement si $\Phi_{\mathfrak{g}}(\mu) \neq 0$.

The same hypothesis is missing in Theorem 13(v) of [1].

2. In some places the symbol $\mathbb{K}$ must be replaced by the symbol $\mathbb{K}^{\times}$as follows:

Everywhere in Sections 3.7 and $3.11 \mathrm{read} \nu \in \mathbb{K}^{\times}$instead of $\nu \in \mathbb{K}$. In the fourth paragraph of Section 3.11 read $\boldsymbol{u}_{\mathfrak{g}, \text { red }}^{*}=\mathbb{K}^{\times} z^{*}$ instead of $\mathfrak{z}_{\mathfrak{g}, \text { red }}^{*}=\mathbb{K} z^{*}$. Everywhere in the tenth and eleventh paragraph of Section 4.4 read $t \in \mathbb{K}^{\times}$instead of $t \in \mathbb{K}$.

DOI: $10.1007 / \mathrm{s} 00031-013-9213-1$

Received December 26, 2012. Accepted December 26, 2012.

Published online February 8, 2013.

* The online version of the original article can be found at http://dx.doi.org/ 10.1007/s00031-012-9179-4. 
288 MICHEL DUFLO, MOHAMED SALAH KHALGUI, PIERRE TORASSO

Aknowledgements. We thank Meher Bouheni for having drawn our attention to the omission of the hypothesis $\operatorname{dim} \mathfrak{z} \leq 1$.

\section{References}

[1] M. Duflo, M. S. Khalgui, P. Torasso, Algèbres de Lie quasi-réductives, Tranform. Groups 17 (2012), no. 2, 417-470. 